\section{Consumo de alimentos ultraprocessados reduz a qualidade global da dieta de gestantes}

\author{
The consumption of ultra-processed foods \\ reduces overall quality of diet in \\ pregnant women
}

\section{El consumo de alimentos ultraprocesados reduce la calidad global de la dieta de gestantes}

Nayara Gomes Graciliano 1

Jonas Augusto Cardoso da Silveira 1

Alane Cabral Menezes de Oliveira 1

\section{Resumo}

Este trabalho analisou a contribuição dos alimentos ultraprocessados (AUP) no perfil alimentar e nutricional da dieta de gestantes. Trata-se de um estudo transversal conduzido com uma amostra representativa de gestantes usuárias de unidades básicas de saúde de Maceió, Alagoas, Brasil. O consumo alimentar foi avaliado pela aplicação de dois recordatórios de 24 horas em dias não consecutivos e os itens de consumo agrupados segundo a classificação NOVA. As estimativas gerais foram expressas no consumo alimentar absoluto (média de ingestão calórica) e relativo (percentual da ingestão energética total segundo grupos de alimentos e itens de consumo). Análises de variâncias foram utilizadas para comparar as médias do consumo energético e de nutrientes, segundo grupos alimentares. A associação entre os quintis de contribuição energética dos AUP (variável de exposição) e (1) itens de consumo e grupos alimentares, (2) contribuição percentual para o total de energia de macronutrientes e (3) densidade de micronutrientes foi analisada por meio de modelos ajustados de regressão linear. O consumo médio de energia das gestantes foi de $1.966,9 \mathrm{Kcal} /$ dia, sendo $22 \%$ proveniente dos AUP. Observou-se relação direta entre a contribuição energética dos AUP na dieta e o consumo energético total $(\beta=228,78 \mathrm{Kcal} ; \mathrm{EP}=21,26)$. Ainda, o aumento da participação de AUP implicou a redução estatisticamente significativa da ingestão de proteínas, fibras, magnésio, ferro, pótassio, zinco, selênio, folato e vitaminas $D$ e E, assim como o consumo de alimentos tradicionais, como arroz, feijão, raízes e tubérculos. Portanto, nossos dados apontam que o consumo de AUP reduz a qualidade global (nutricional e alimentar) da dieta de gestantes.

Alimentos Industrializados; Qualidade dos Alimentos; Consumo de Alimentos; Gestantes; Epidemiologia Nutricional

\author{
Correspondência \\ A. C. M. Oliveira \\ Faculdade de Nutrição, Universidade Federal de Alagoas, \\ Av. Lourival de Melo Mota s/n, Maceió, AL 57072-900, Brasil. \\ alanecabral@gmail.com \\ 1 Faculdade de Nutrição, Universidade Federal de Alagoas, \\ Maceió, Brasil.
}




\section{Introdução}

A gestação é um período marcado por grandes modificações fisiológicas e metabólicas, as quais contribuem para o aumento da vulnerabilidade a inadequações relacionadas à ingestão dietética $\mathrm{e}$ ao estado nutricional ${ }^{1}$. Nesse aspecto, as mudanças são refletidas especialmente no aumento das demandas de alguns micronutrientes, como ferro, ácido fólico e zinco, e no aumento das necessidades energéticas que variam em função do estado nutricional pré-gestacional. Portanto, trata-se de um período fundamental para a promoção e manutenção de uma alimentação adequada e saudável, uma vez que impactará diretamente na saúde do binômio mãe/filho 2 .

Do ponto de vista epidemiológico, as mudanças no padrão alimentar e nutricional ocorridas nas últimas décadas são representadas pela redução do consumo de frutas, hortaliças, cereais e leguminosas e aumento da ingestão de alimentos com alta densidade energética, ricos em açúcares, gorduras e sódio 3,4. Portanto, esse mesmo padrão que se coloca como risco para doenças crônicas não transmissíveis (DCNT) também representa uma importante ameaça no período gestacional, bem como para as condições de saúde no início da vida dos indivíduos 5 .

Estudos têm demonstrado que uma nutrição adequada durante a gestação é fundamental para a prevenção de desfechos gestacionais negativos 6,7. Por outro lado, o consumo de ultraprocessados está associado à obesidade materna 8 , aumento do ganho de peso gestacional e da gordura corporal neonatal 9 que podem aumentar o risco para parto cesáreo, macrossomia fetal e nascimentos de recém-nascidos grandes para a idade gestacional (GIG) 10. Assim, o monitoramento do estado nutricional e do padrão alimentar de gestantes tem sido reconhecido como uma estratégia prioritária para a promoção e a proteção da saúde materno-infantil 11.

A aplicação da classificação NOVA em estudos de consumo alimentar tem sido utilizada com sucesso para descrever padrões alimentares, avaliar mudanças no consumo de alimentos ultraprocessados (AUP) ao longo do tempo e analisar a associação entre a contribuição destes alimentos com o perfil nutricional das dietas e com os resultados de saúde 5 . Referem-se a formulações industriais cuja fabricação envolve diferentes etapas e técnicas de processamento, além de vários ingredientes que em muitos casos são sintetizados apenas em laboratório e de uso exclusivo da indústria 12,13.

No Brasil, apesar da associação entre o impacto de AUP na qualidade da dieta e sobre o estado nutricional já terem sido relatados para população em geral 3,5,14,15, a disponibilidade de informação sobre o consumo alimentar de gestantes ainda é escassa, especialmente considerando a adesão às recomendações do Guia Alimentar para a População Brasileira 12 e as regionalidades do país. Assim, este trabalho teve por objetivo analisar o perfil nutricional da dieta de gestantes provenientes da atenção primária à saúde no Município de Maceió, Alagoas, com base na estrutura teórica da classificação NOVA.

\section{Metodologia}

Trata-se de um estudo transversal realizado no Município de Maceió entre setembro de 2013 e fevereiro de 2014. A identificação das gestantes atendidas nas unidades básicas de saúde (UBS) da rede pública foi realizada por meio de amostragem probabilística estratificada em dois estágios. Inicialmente, foram sorteadas 50\% das UBS do município, segundo a distribuição proporcional das unidades nos oito Distritos Sanitários. Em seguida, calculou-se o número proporcional de gestantes a serem arroladas no estudo de acordo com o número de gestantes cadastradas em cada UBS sorteada na etapa anterior.

O cálculo da amostra para a população finita $(\mathrm{n}=8.949)$ foi feito no programa Epi Info 7 (https:// www.cdc.gov/epiinfo/index.html), considerando-se a frequência antecipada do desfecho primário da pesquisa, o excesso de peso em gestantes, estimado em $27,5 \% 16$, adotando-se intervalo de $95 \%$ de confiança (IC95\%), margem de erro de 5\% e acréscimo de 10\% (para perdas amostras), resultando em um total de 322 gestantes.

Foram incluídas gestantes de feto único, residentes em Maceió e atendidas pela rede pública municipal. Os critérios de exclusão foram: alterações físicas que pudessem distorcer a avaliação antropométrica e a presença de problemas neurológicos. Para as análises foram excluídas as gestantes 
que não tinham dados de dois recordatórios de 24 horas (R24h) ou que apresentavam informações incompletas sobre o tamanho das porções, quantidades e/ou volumes consumidos, assim como o tipo de preparação. A amostra final foi composta por 295 gestantes (8,4\% de perdas).

A coleta dos dados socioeconômicos, clínicos, demográficos e dietéticos foi realizada em questionários semiestruturados por nutricionistas treinadas. A antropometria (peso e altura) foi realizada com balança digital da marca Marte LC200 (Marte Científica, São Paulo, Brasil) e estadiômetro portátil Wood (WCS Tecnologia, Curitiba, Brasil). A classificação do estado nutricional das gestantes, baseada no índice de massa corporal (IMC), foi realizada segundo Atalah et al. 17 e o ganho ponderal gestacional de acordo com as recomendações do Instituto de Medicina dos Estados Unidos 18.

Os dados de consumo alimentar foram coletados pela aplicação de dois R24h, em dias não consecutivos. Foram registrados o tipo de alimento, a forma de consumo, o nome da preparação e, sempre que possível, os ingredientes utilizados e a forma de preparo, assim como a quantidade consumida em medidas caseiras. O primeiro $\mathrm{R} 24 \mathrm{~h}$ foi realizado durante a entrevista no pré-natal e o segundo por telefone, com intervalo de até um mês após a coleta inicial. Para a análise do consumo alimentar, a quantidade de cada alimento foi transformada em gramas ou mililitros com o auxílio de tabelas de medidas caseiras de alimentos e preparações 19,20.

O cálculo nutricional dos R24h foi realizado com base na tabela de composição nutricional dos alimentos consumidos no Brasil, usada na Pesquisa de Orçamentos Familiares (POF) 2008-2009. Quando algum alimento ou preparação não foi encontrado na tabela optou-se por considerar um alimento/ preparação padrão equivalente, compatível com as características nutricionais do alimento ou preparação original. Quando não informada, a quantidade de adição de açúcar em sucos de fruta, café, chá e leite foi padronizada em 10\% do volume consumido, seguindo o critério adotado pelo Instituto Brasileiro de Geografia e Estatística (IBGE) 21.

Os itens de consumo foram classificados segundo os quatro grupos alimentares da classificação NOVA: (1) alimentos in natura ou minimamente processados; (2) ingredientes culinários; (3) alimentos processados; e (4) AUP 13. As preparações alimentares que não foram desagregadas em seus ingredientes constituintes durante a aplicação do R24h foram incluídas no estudo como preparações culinárias e classificadas de acordo com as características de seus ingredientes principais. Assim, por exemplo, "pizza de muçarela" foi listada como pertencente ao Grupo 3 e "enroladinho de salsicha" como preparação culinária do Grupo 4 (Material Suplementar. Quadro S1. http://cadernos.ensp.fiocruz.br/ static//arquivo/suppl-e00030120-pt_9463.pdf).

A correção da variabilidade intraindividual foi realizada pelo cálculo da média de consumo observado nos dois R24h aplicados. Estatísticas descritivas foram usadas para analisar o consumo alimentar absoluto (média de ingestão calórica) e relativo (percentual da ingestão energética total segundo grupos de alimentos e itens de consumo) das gestantes. Análises de variâncias com teste post hoc de Bonferroni foram utilizadas a fim de comparar as médias do consumo energético e de nutrientes, segundo grupos alimentares (Material Suplementar. Tabela S1. http://cadernos.ensp.fiocruz.br/static// arquivo/suppl-e00030120-pt_9463.pdf).

Em seguida, as gestantes foram estratificadas em cinco grupos, correspondentes aos quintis de consumo energético médio proveniente dos alimentos ultraprocessados, ou seja, energia fornecida pelo Grupo 4. Essa estratificação foi tratada em todos os modelos como uma variável de exposição ordinal.

A associação entre os quintis de consumo provenientes de AUP e a distribuição percentual da ingestão total de energia por grupos de alimentos e itens de consumo, bem como a ingestão total de energia e de nutrientes, foram analisadas por meio de modelos de regressão linear multivariável.

Modelos lineares generalizados (família binomial e função de ligação $\log$ ) foram utilizados para estimar a razão de prevalências (RP) de inadequação da ingestão em relação aos quintis de consumo energético dos ultraprocessados. Pela ausência de recomendações específicas para gestantes com base na densidade de nutrientes, utilizou-se as metas nutricionais da Organização Mundial da Saúde (OMS) para a prevenção de DCNT 22,23 a fim de avaliar a prevalência de ingestão inadequada de açúcares livres, gorduras totais, saturadas e trans, fibra, sódio e potássio, segundo quintis da contribuição de energia proveniente dos AUP. Essa decisão foi baseada nos riscos associados com o ganho de peso gestacional excessivo, diabetes gestacional, pré-eclâmpsia e macrossomia e alterações epigenéticas fetais 1,24 . 
Todas as análises foram ajustadas para idade e escolaridade materna, recebimento de benefício do governo e trabalho fora do lar, selecionadas com base no referencial teórico da influência no consumo alimentar 2,6,11,21. Por tratar-se de fatores considerados importantes na literatura para o ajuste das estimativas, forçou-se a entrada das variáveis nos modelos.

O método da densidade de nutrientes foi usado para o ajuste de ingestão total de energia para as variáveis de desfecho. Os coeficientes de regressão foram apresentados em sua escala original, com seus respectivos erros-padrão (EP) e valor de p, ou como RP e IC95\%.

Todos os testes foram realizados no software Stata/MP 13.0 (https://www.stata.com), considerando $\mathrm{p}<0,05$ como nível de significância estatística.

Este trabalho faz parte do projeto Estado Nutricional, Ganho Ponderal e Comportamento Alimentar de Gestantes de Maceió - Alagoas: Impacto Sobre a Saúde do Binômio Mãe-Filho, financiado pela chamada FAPEAL/CNPq Projeto de Pesquisa para o Sistema Único de Saúde (processo 60030.000741/2013) e aprovado pelo Comitê de Ética e Pesquisa da Universidade Federal de Alagoas (CAAE: 18807113.3.0000.5013).

\section{Resultados}

A amostra analítica incluiu 295 gestantes de 14 a 43 anos, com idade média de 23,7 anos (DP \pm 5,9). Cerca de 3/4 delas eram casadas ou estavam em união estável, 76,8\% se autodeclararam negras ou pardas e 52,2\% e 60,9\% tinham, respectivamente, até 9 anos de estudos e renda familiar menor ou igual a um salário mínimo. Sobre a idade gestacional, 45,8\% da amostra estavam no segundo trimestre de gravidez. Em relação ao estado nutricional pré-gestacional, 67\% iniciaram a gravidez eutróficas, porém, apenas 40,5\% apresentaram ganho de peso gestacional adequado (Tabela 1 ).

\section{Tabela 1}

\begin{tabular}{|c|c|c|}
\hline Variáveis & $\mathbf{n}$ & $\%$ \\
\hline \multicolumn{3}{|c|}{ Idade materna (anos) } \\
\hline$\leq 19$ & 75 & 25,4 \\
\hline $20-34$ & 200 & 67,8 \\
\hline$\geq 35$ & 20 & 6,8 \\
\hline \multicolumn{3}{|c|}{ Cor da pele autorreferida } \\
\hline Branca & 67 & 23,2 \\
\hline Parda & 176 & 60,9 \\
\hline Negra & 46 & 15,9 \\
\hline \multicolumn{3}{|l|}{ Situação conjugal } \\
\hline Solteira & 63 & 21,6 \\
\hline União estável & 229 & 78,4 \\
\hline \multicolumn{3}{|l|}{ Anos de estudo } \\
\hline$\leq 9$ & 154 & 52,2 \\
\hline $10-12$ & 127 & 43,1 \\
\hline$\geq 13$ & 14 & 4,7 \\
\hline \multicolumn{3}{|c|}{ Trabalha fora do lar } \\
\hline Sim & 83 & 28,1 \\
\hline Não & 212 & 71,9 \\
\hline \multicolumn{3}{|c|}{ Renda familiar (salários mínimos) } \\
\hline$\leq 1$ & 176 & 60,9 \\
\hline$>1$ & 113 & 39,1 \\
\hline
\end{tabular}

(continua) 
Tabela 1 (continuação)

\begin{tabular}{|c|c|c|}
\hline Variáveis & $\mathbf{n}$ & $\%$ \\
\hline \multicolumn{3}{|c|}{ Número de membros na família } \\
\hline$>5$ & 32 & 10,8 \\
\hline$\leq 5$ & 263 & 89,2 \\
\hline \multicolumn{3}{|l|}{ Linha da pobreza } \\
\hline Sim & 10 & 3,5 \\
\hline Não & 279 & 96,5 \\
\hline \multicolumn{3}{|c|}{ Beneficiária de programa governamental } \\
\hline Sim & 131 & 44,4 \\
\hline Não & 164 & 55,6 \\
\hline \multicolumn{3}{|l|}{ Acesso à água encanada } \\
\hline Sim & 291 & 98,6 \\
\hline Não & 4 & 1,4 \\
\hline \multicolumn{3}{|l|}{ Tabagismo } \\
\hline Sim & 28 & 9,5 \\
\hline Não & 267 & 90,5 \\
\hline \multicolumn{3}{|l|}{ Primigesta } \\
\hline Sim & 120 & 4,7 \\
\hline Não & 175 & 59,3 \\
\hline \multicolumn{3}{|c|}{ Idade gestacional (semanas) } \\
\hline 1 ㅇ trimestre (3-13) & 47 & 15,9 \\
\hline 2o trimestre (14-26) & 135 & 45,8 \\
\hline 3o trimestre (27-42) & 113 & 38,3 \\
\hline \multicolumn{3}{|l|}{ IMC pré-gestacional } \\
\hline Baixo peso & 26 & 9,3 \\
\hline Eutrofia & 87 & 67,0 \\
\hline Sobrepeso & 42 & 15,1 \\
\hline Obesidade & 24 & 8,6 \\
\hline \multicolumn{3}{|c|}{ Ganho de peso gestacional } \\
\hline Insuficiente & 98 & 35,1 \\
\hline Adequado & 113 & 40,5 \\
\hline Excessivo & 68 & 24,4 \\
\hline \multicolumn{3}{|l|}{ IMC gestacional } \\
\hline Baixo peso & 50 & 16,9 \\
\hline Adequado & 134 & 45,9 \\
\hline Sobrepeso & 79 & 26,8 \\
\hline Obesidade & 32 & 10,9 \\
\hline
\end{tabular}

IMC: índice de massa corporal.

A análise dos R24h apontou que o consumo médio de energia foi de 1.966,9 Kcal/dia, sendo 56,7\% provenientes de alimentos in natura ou minimamente processados, 9,7\% de ingredientes culinários, $11,4 \%$ de alimentos processados e 22,2\% de produtos ultraprocessados (Tabela 2).

No grupo de alimentos in natura ou minimamente processados, os que mais contribuíram com o consumo energético total foram as carnes bovina, de porco e de aves (13,4\%), as frutas (8,8\%), arroz, massa e farinhas (6,8\% para cada um deles) e feijão (6,4\%). É importante destacar que no grupo de frutas foram incluídos também os sucos (da fruta ou de polpa) que possivelmente elevaram a contribuição energética deste subgrupo de alimentos. No grupo de massa e farinhas também foi incluída a farinha de milho, que é um ingrediente bastante consumido no Nordeste do país sob a forma de cuscuz. Com menor contribuição energética aparecem outros cereais e grãos, verduras/legumes e vísceras (Tabela 2). 
Tabela 2

Médias do consumo absoluto e relativo de alimentos in natura ou minimamente processados, de ingredientes culinários, de alimentos processados e de alimentos ultraprocessados das gestantes atendidas na rede básica de saúde do Município de Maceió, Alagoas, Brasil, 2013-2014 (N = 295).

\begin{tabular}{|c|c|c|}
\hline Grupo de alimentos e itens de consumo & Kcal/dia & $\begin{array}{l}\text { Ingestão total } \\
\text { de energia (\%) }\end{array}$ \\
\hline Alimentos in natura ou minimamente processados & $1.102,9$ & 56,7 \\
\hline Carne bovina, suína e de aves & 263,4 & 13,4 \\
\hline Frutas * & 171,8 & 8,8 \\
\hline Arroz & 125,9 & 6,8 \\
\hline Massa e farinhas ** & 142,8 & 6,8 \\
\hline Feijão & 122,5 & 6,4 \\
\hline Raízes e tubérculos & 76,3 & 3,8 \\
\hline Preparações culinárias *** & 49,6 & 2,7 \\
\hline Leite e iogurte natural & 46,9 & 2,5 \\
\hline Ovos & 32,7 & 1,7 \\
\hline Café e chás & 29,1 & 1,5 \\
\hline Peixes e frutos do mar & 24,1 & 1,3 \\
\hline Vísceras & 12,5 & 0,7 \\
\hline Verduras e legumes & 4,0 & 0,2 \\
\hline Outros cereais e grãos \# & 1,3 & 0,1 \\
\hline Ingredientes culinários & 191,6 & 9,7 \\
\hline Açúcar de adição e mel & 174,5 & 8,8 \\
\hline Manteiga & 17,1 & 0,9 \\
\hline Alimentos processados & 221,8 & 11,4 \\
\hline Pão & 166,7 & 8,7 \\
\hline Carnes salgadas & 36,9 & 1,8 \\
\hline Queijos & 10,0 & 0,5 \\
\hline Preparações culinárias à base de alimentos processados & 6,2 & 0,3 \\
\hline Conservas & 2,0 & 0,1 \\
\hline Alimentos ultraprocessados & 450,6 & 22,2 \\
\hline Biscoitos doces, bolachas salgadas e salgadinhos do tipo chips & 193,1 & 9,5 \\
\hline Doces, bolos e tortas & 49,4 & 2,4 \\
\hline Embutidos & 44,3 & 2,3 \\
\hline Refrigerantes e bebidas adoçadas & 43,3 & 2,2 \\
\hline Preparações culinárias à base de alimentos ultraprocessados & 41,1 & 2,0 \\
\hline Bebidas lácteas adoçadas & 34,7 & 1,7 \\
\hline Margarina & 23,4 & 1,0 \\
\hline Pratos prontos ou semiprontos \#\# & 19,7 & 1,0 \\
\hline Molhos & 1,6 & 0,1 \\
\hline Total & $1.966,9$ & 100,0 \\
\hline
\end{tabular}

* Incluem sucos de fruta e de polpa de frutas;

** Farinha de mandioca, farinha de milho, farinha de tapioca e macarrão;

*** À base de alimentos in natura ou minimamente processados;

\# Aveia, linhaça, granola e castanha do Pará;

\#\# Batata palito congelada, hambúrguer, lasanha congelada, empanado de frango congelado e macarrão instantâneo. 
Quanto aos ingredientes culinários, apenas três alimentos puderam ser avaliados devido à disponibilidade dos dados nos R24h. O açúcar de adição, normalmente colocado em bebidas como café, chás e sucos, contribuiu com 8,8\% do total das calorias ingeridas. Dentre os alimentos processados, o pão foi o principal responsável pela contribuição energética do grupo $(8,7 \%)$.

Dos alimentos que compõem o grupo dos ultraprocessados, os biscoitos doces e salgados e os salgadinhos do tipo chips contribuíram com 9,5\% da energia média ingerida, sendo o segundo maior item de consumo em termos porcentuais da ingestão energética total da alimentação das gestantes (Tabela 2).

Na Tabela 3, é apresentada a contribuição percentual de energia média dos grupos de alimentos e itens de consumo, segundo quintis de consumo de energia provinda dos AUP. Nessas análises, observou-se associação inversa e estatisticamente significativa com alimentos in natura e minimamente processados, como o arroz, feijão, carnes, ovos, raízes e tubérculos, frutas, verduras e legumes. Em relação ao padrão de consumo de ultraprocessados, observou-se que a contribuição percentual

\section{Tabela 3}

Distribuição (\%) da ingestão total de energia segundo grupos de alimentos e itens de consumo em estratos das gestantes atendidas na rede básica de saúde do Município de Maceió, Alagoas, Brasil, correspondente a quintis (Q1, Q2, Q3, Q4, Q5) do consumo de alimentos ultraprocessados, 2013-2014 ( $=295)$.

\begin{tabular}{|c|c|c|c|c|c|c|c|}
\hline \multirow[t]{2}{*}{ Grupo de alimentos e itens de consumo } & \multicolumn{5}{|c|}{$\begin{array}{l}\text { Quintis de consumo energético proveniente de } \\
\text { alimentos ultraprocessados } \\
\text { (\% do total de energia) }\end{array}$} & \multirow[t]{2}{*}{$\beta(E P)$} & \multirow[t]{2}{*}{ Valor de $p$ * } \\
\hline & Q1 ** & Q2 ** & Q3 ** & Q4 ** & Q5 ** & & \\
\hline Alimentos in natura ou minimamente processados & 74,4 & 62,7 & 53,4 & 51,2 & 41,7 & $-7,71(0,50)$ & $<0,001$ \\
\hline Arroz & 9,8 & 7,3 & 6,8 & 5,9 & 4,3 & $-1,19(0,23)$ & $<0,001$ \\
\hline Feijão & 7,3 & 7,4 & 6,1 & 6,0 & 5,1 & $-0,53(0,19)$ & 0,006 \\
\hline Carne bovina, suína e de aves & 20,0 & 14,2 & 12,1 & 12,3 & 8,3 & $-2,62(0,38)$ & $<0,001$ \\
\hline Vísceras & 1,0 & 0,8 & 0,6 & 0,5 & 0,5 & $-0,13(0,09)$ & 0,178 \\
\hline Ovos & 2,7 & 1,9 & 1,4 & 1,5 & 1,2 & $-0,33(0,11)$ & 0,005 \\
\hline Peixes e frutos do mar & 1,5 & 1,2 & 1,1 & 1,5 & 1,0 & $-0,03(0,15)$ & 0,824 \\
\hline Raízes e tubérculos & 6,2 & 4,1 & 3,0 & 3,1 & 2,4 & $-0,85(0,26)$ & 0,002 \\
\hline Massa e farinhas *** & 6,2 & 8,4 & 6,0 & 6,9 & 6,7 & $-0,13(0,30)$ & 0,666 \\
\hline Leite e iogurte natural & 3,3 & 2,0 & 2,7 & 1,9 & 2,6 & $-0,15(0,17)$ & 0,388 \\
\hline Café e chás & 1,2 & 2,0 & 2,0 & 1,2 & 1,0 & $-0,11(0,07)$ & 0,123 \\
\hline Frutas \# & 10,8 & 10,1 & 8,8 & 7,9 & 6,4 & $-1,09(0,26)$ & $<0,001$ \\
\hline Verduras e legumes & 0,4 & 0,3 & 0,2 & 0,2 & 0,1 & $-0,07(0,02)$ & 0,002 \\
\hline Outros cereais e grãos \#\# & 0,0 & 0,2 & 0,1 & 0,0 & 0,0 & $-0,01(0,02)$ & 0,592 \\
\hline Preparações culinárias \#\#\# & 4,0 & 2,8 & 2,5 & 2,3 & 2,1 & $-0,41(0,20)$ & 0,040 \\
\hline Ingredientes culinários & 9,1 & 10,9 & 10,7 & 9,5 & 8,1 & $-0,40(0,21)$ & 0,059 \\
\hline Açúcar de adição e mel & 8,6 & 9,5 & 9,7 & 8,6 & 7,5 & $-0,33(0,20)$ & 0,098 \\
\hline Manteiga & 0,5 & 1,4 & 1,0 & 0,9 & 0,6 & $-0,06(0,07)$ & 0,359 \\
\hline Alimentos processados & 11,6 & 11,5 & 14,3 & 9,7 & 10,1 & $-0,48(0,39)$ & 0,221 \\
\hline Carnes salgadas & 1,3 & 1,4 & 2,7 & 1,2 & 2,2 & $0,23(0,21)$ & 0,276 \\
\hline Conservas & 0,3 & 0,1 & 0,0 & 0,1 & 0,0 & $-0,04(0,02)$ & 0,117 \\
\hline Queijos & 0,5 & 0,6 & 0,7 & 0,3 & 0,5 & $-0,01(0,06)$ & 0,796 \\
\hline Pães & 9,3 & 9,3 & 10,3 & 7,9 & 7,0 & $-0,70(0,32)$ & 0,030 \\
\hline Preparações culinárias § & 0,2 & 0,1 & 0,6 & 0,2 & 0,4 & $0,04(0,07)$ & 0,493 \\
\hline
\end{tabular}

(continua) 
Tabela 3 (continuação)

\begin{tabular}{|c|c|c|c|c|c|c|c|}
\hline \multirow[t]{2}{*}{ Grupo de alimentos e itens de consumo } & \multicolumn{5}{|c|}{$\begin{array}{c}\text { Quintis de consumo energético proveniente de } \\
\text { alimentos ultraprocessados } \\
\text { ( } \% \text { do total de energia) }\end{array}$} & \multirow[t]{2}{*}{$\beta(E P)$} & \multirow[t]{2}{*}{ Valor de $p$ * } \\
\hline & Q1 ** & $\mathrm{Q} 2$ ** & Q3 ** & Q4 ** & Q5 ** & & \\
\hline Alimentos ultraprocessados & 4,9 & 14,9 & 21,6 & 29,6 & 40,1 & $8,60(0,32)$ & $<0,001$ \\
\hline Refrigerantes & 0,5 & 1,3 & 2,7 & 2,4 & 4,1 & $0,84(0,13)$ & $<0,001$ \\
\hline Embutidos & 1,1 & 2,0 & 2,3 & 2,0 & 3,8 & $0,52(0,16)$ & 0,001 \\
\hline Margarina & 0,4 & 0,6 & 2,0 & 1,0 & 1,3 & $0,21(0,12)$ & 0,086 \\
\hline Bebidas lácteas adoçadas & 0,3 & 1,2 & 0,9 & 3,6 & 2,8 & $0,74(0,15)$ & $<0,001$ \\
\hline Pratos prontos ou semiprontos §§ & 0,1 & 0,8 & 1,0 & 2,2 & 1,0 & $0,34(0,13)$ & 0,010 \\
\hline Molhos & 0,0 & 0,0 & 0,0 & 0,3 & 0,1 & $0,05(0,03)$ & 0,072 \\
\hline Doces, bolos e tortas & 0,5 & 1,3 & 2,4 & 3,0 & 4,7 & $1,06(0,19)$ & $<0,001$ \\
\hline Biscoitos §§§ & 1,7 & 6,3 & 8,4 & 12,9 & 18,1 & $3,94(0,33)$ & $<0,001$ \\
\hline Preparações culinárias † & 0,3 & 1,4 & 1,9 & 2,2 & 4,2 & $0,86(0,16)$ & $<0,001$ \\
\hline Total & 100,0 & 100,0 & 100,0 & 100,0 & 100,0 & - & - \\
\hline
\end{tabular}

EP: erro padrão.

* Obtido por modelo de regressão linear múltipla ajustado para idade, recebimento de benefício do governo, escolaridade e trabalho fora do lar;

** Médias e intervalos de 95\% de confiança de dos quintis de ingestão energética proveniente de alimentos ultraprocessados: Q1 = 72,4Kcal (59,7-85,1);

Q2 = 245,1 Kcal (231,8-258,5); Q3 = 398,9Kcal (389,2-408,6); Q4 = 583,4Kcal (565,3-601,5); Q5 = 954,8Kcal (894,9-1.010,7);

*** Farinha de mandioca, farinha de milho, farinha de tapioca e macarrão;

\# Incluem sucos de fruta e de polpa de frutas;

\#\# Aveia, linhaça, granola e castanha do Pará;

\#\#\# À base de alimentos in natura ou minimamente processados;

$\S$ À base de alimentos processados;

§§ Batata palito congelada, hambúrguer, lasanha congelada, empanado de frango congelado e macarrão instantâneo;

$\S \S \S$ Biscoitos doces, bolachas salgadas e salgadinhos do tipo chips;

† À base de alimentos ultraprocessados.

energética do quintil superior foi 8,2 vezes maior que o primeiro; em termos absolutos, a diferença média na ingestão energética das gestantes entre o primeiro e o quinto quintis foi $882,4 \mathrm{Kcal}$. Por fim, a contribuição energética de alimentos in natura e minimamente processados em relação aos ultraprocessados dentre as mulheres no último quintil foi, praticamente, 1:1.

Com exceção da margarina e dos molhos, todas as categorias de AUP aumentaram significativamente do primeiro para o último quintil. Tendência inversa foi verificada para o consumo de pão no grupo de alimentos processados. Para as demais categorias de alimentos não houve variação significativa em relação aos quintis de consumo de produtos ultraprocessados (Tabela 3).

A dinâmica em relação à contribuição energética dos grupos de alimentos e itens de consumo, segundo quintis de consumo de energia provinda dos AUP (Tabela 3), também é refletida nos indicadores nutricionais do consumo alimentar (Material Suplementar. Tabela S1. http://cadernos.ensp. fiocruz.br/static//arquivo/suppl-e00030120-pt_9463.pdf). Em relação à distribuição dos macronutrientes, a fração de AUP, quando comparada à fração da dieta relativa aos alimentos in natura ou minimamente processados, tem quase o dobro de açúcar livre, maior teor de gorduras em geral, incluindo a saturada, e oito vezes mais gordura trans. Esse padrão também é observado em relação à diferença média na ingestão de micronutrientes: potássio $(1.131 \mathrm{mg})$, ferro $(3 \mathrm{mg})$, selênio $(39,5 \mathrm{mcg})$, magnésio (90,4mg), vitamina A $(771,9 \mathrm{mcg})$, vitamina C $(1.070,2 \mathrm{mg})$ e folato $(141,2 \mathrm{mcg})$ (Material Suplementar. Tabela S1. http://cadernos.ensp.fiocruz.br/static//arquivo/suppl-e00030120-pt_9463.pdf).

Nas análises envolvendo os indicadores do perfil nutricional da dieta segundo os quintis de contribuição energética dos AUP (Tabela 4), identificou-se que houve uma relação exposição-resposta positiva e estatisticamente significativa entre a ingestão energética total e o percentual de contribuição energética proveniente dos AUP $(228,78 \mathrm{Kcal}$; EP = 21,26); na comparação entre o primeiro e o último quintis, observa-se que houve uma diferença média de 962,2Kcal (163\%). Além da ingestão energética, 
Médias de indicadores nutricionais do consumo alimentar de gestantes atendidas na rede básica de saúde do Município de Maceió, Alagoas, Brasil, correspondentes a quintis (Q1, Q2, Q3, Q4, Q5) do consumo de alimentos ultraprocessados, 2013-2014 (N = 295).

\begin{tabular}{|c|c|c|c|c|c|c|c|}
\hline \multirow[t]{2}{*}{ Indicador } & \multicolumn{5}{|c|}{$\begin{array}{l}\text { Quintil de consumo de alimentos } \\
\text { ultraprocessados ( } \% \text { do total de energia) }\end{array}$} & \multirow[t]{2}{*}{$\beta(E P)$} & \multirow[t]{2}{*}{ Valor de $p$ * } \\
\hline & Q1 & Q2 & Q3 & Q4 & Q5 & & \\
\hline Total de energia (Kcal/dia) & $1.532,5$ & $1.733,9$ & $1.932,3$ & $2.141,4$ & $2.494,7$ & $228,78(21,26)$ & $<0,001$ \\
\hline \multicolumn{8}{|c|}{ Contribuição percentual para o total de energia de } \\
\hline Proteínas & 19,1 & 16,7 & 15,4 & 15,2 & 13,9 & $-1,15(0,16)$ & $<0,001$ \\
\hline Carboidratos & 56,6 & 59,1 & 57,7 & 57,6 & 58,8 & $0,26(0,34)$ & 0,440 \\
\hline Açúcares livres & 20,9 & 23,7 & 25,2 & 25,7 & 28,0 & $1,60(0,33)$ & $<0,001$ \\
\hline Gorduras totais & 24,9 & 24,8 & 27,1 & 27,0 & 27,6 & $0,77(0,24)$ & 0,002 \\
\hline Gordura saturada & 8,4 & 8,6 & 9,6 & 9,2 & 9,6 & $0,29(0,10)$ & 0,005 \\
\hline Gordura trans & 0,9 & 1,1 & 1,8 & 1,8 & 2,3 & $0,33(0,04)$ & $<0,001$ \\
\hline \multicolumn{8}{|l|}{ Densidade de nutrientes } \\
\hline Fibra (g/1.000Kcal) & 10,1 & 9,7 & 8,6 & 8,5 & 7,8 & $-0,55(0,11)$ & $<0,001$ \\
\hline Cálcio (mg/1.000Kcal) & 248,1 & 239,2 & 244,8 & 242,5 & 244,2 & $-0,13(4,74)$ & 0,977 \\
\hline Magnésio (mg/1.000Kcal) & 132,8 & 127,7 & 113,3 & 116,0 & 110,1 & $-5,40(1,00)$ & $<0,001$ \\
\hline Ferro (mg/1.000Kcal) & 5,6 & 5,5 & 5,0 & 4,9 & 5,0 & $-0,15(0,06)$ & 0,011 \\
\hline Sódio (mg/1.000Kcal) & $1.676,8$ & $1.599,5$ & $1.586,6$ & $1.472,2$ & $1.472,8$ & $-51,22(14,66)$ & 0,001 \\
\hline Potássio (mg/1.000Kcal) & $1.365,4$ & $1.180,8$ & $1.086,0$ & $1.068,3$ & 988,7 & $-83,72(11,15)$ & $<0,001$ \\
\hline Cobre (mg/1.000Kcal) & 1,2 & 1,0 & 0,9 & 0,8 & 0,8 & $-0,10(0,06)$ & 0,140 \\
\hline Zinco (mg/1.000Kcal) & 5,9 & 5,3 & 5,1 & 4,7 & 4,5 & $-0,35(0,06)$ & $<0,001$ \\
\hline Selênio (mcg/1.000Kcal) & 48,5 & 47,2 & 37,9 & 40,8 & 35,2 & $-3,23(0,84)$ & $<0,001$ \\
\hline Vitamina A (mcg/1.000Kcal) & 849,8 & 569,7 & 555,5 & 449,7 & 469,4 & $-84,86(46,82)$ & 0,071 \\
\hline Folato (mcg/1.000Kcal) & 126,3 & 132,8 & 111,2 & 107,2 & 104,0 & $-6,71(2,11)$ & 0,002 \\
\hline Vitamina D (mcg/1.000Kcal) & 1,7 & 1,4 & 1,4 & 1,4 & 1,3 & $-0,08(0,03)$ & 0,031 \\
\hline Vitamina E (mg/1.000Kcal) & 2,2 & 1,8 & 1,7 & 1,7 & 1,5 & $-0,14(0,02)$ & $<0,001$ \\
\hline Vitamina C (mg/1.000Kcal) & 546,6 & 553,2 & 466,1 & 465,3 & 499,5 & $-14,30(36,49)$ & 0,695 \\
\hline
\end{tabular}

EP: erro padrão.

* Obtido por modelo de regressão linear múltipla ajustado para idade, recebimento de benefício do governo, escolaridade e trabalho fora do lar.

essa mesma relação foi reproduzida para o consumo de açúcar livre (1,6\%; EP = 0,33), gordura total $(0,77 \%$; $\mathrm{EP}=0,24)$, gordura saturada $(0,29 \%$; $\mathrm{EP}=0,10)$ e gordura trans $(0,33 \% ; \mathrm{EP}=0,04)$.

Relação exposição-resposta inversa foi identificada para proteína $(-1,15 \%$; EP $=0,16)$, fibras $(-0,55 \mathrm{~g} ; \mathrm{EP}=0,11)$, magnésio $(-5,40 \mathrm{mg} ; \mathrm{EP}=1,00)$, ferro $(-0,15 \mathrm{mg}$; $\mathrm{EP}=0,06)$, sódio $(-51,22 \mathrm{mg}$; $\mathrm{EP}=14,66)$, potássio $(-83,72 \mathrm{mg} ; \mathrm{EP}=11,15)$, zinco $(-0,35 \mathrm{mg} ; \mathrm{EP}=0,06)$, selênio $(-3,23 \mathrm{mcg} ; \mathrm{EP}=$ 0,84), folato (-6,71 mcg; EP = 2,11) e vitaminas D (-0,08mcg; EP = 0,03) e E (-0,14mg; EP = 0,02). Para carboidratos, cálcio, cobre e vitaminas A e C não houve variação estatisticamente significativa em relação aos quintis de consumo de ultraprocessados, no entanto, a ingestão de vitamina A no primeiro quintil é quase o dobro da ingestão no último.

A Tabela 5 mostra a prevalência de ingestão inadequada de nutrientes de acordo com os quintis de contribuição energética do consumo de ultraprocessados. De antemão, nota-se que, independentemente da fração de consumo de ultraprocessados, a prevalência de inadequação de nutrientes críticos, segundo as recomendações da OMS, foi muito elevada. No caso das gorduras trans, o aumento da prevalência de inadequação esteve positivamente associado à maior ingestão energética proveniente de ultraprocessados $(\mathrm{RP}=1,54$; IC95\%: 1,37-1,72); a diferença relativa entre o primeiro e o último quintis correspondeu a $240 \%$. 
Tabela 5

Prevalência de ingestão inadequada de nutrientes nos estratos das gestantes atendidas na rede básica de saúde do Município de Maceió, Alagoas, Brasil, correspondente a quintis (Q1, Q2, Q3, Q4, Q5) do consumo de alimentos ultraprocessados, 2013-2014 (N = 295).

\begin{tabular}{|c|c|c|c|c|c|c|c|c|c|}
\hline \multirow[t]{2}{*}{ Indicador } & \multirow{2}{*}{$\begin{array}{c}\text { Valores } \\
\text { recomendados } \\
\text { para os indicadores }\end{array}$} & \multirow[t]{2}{*}{ Total } & \multicolumn{5}{|c|}{$\begin{array}{l}\text { Prevalência de ingestão inadequada de acordo com o } \\
\text { quintil de consumo de alimentos ultraprocessados (\%) }\end{array}$} & \multirow[t]{2}{*}{ RP } & \multirow[t]{2}{*}{ IC95\% } \\
\hline & & & Q1 & Q2 & Q3 & Q4 & Q5 & & \\
\hline \multicolumn{10}{|l|}{$\begin{array}{l}\text { Contribuição percentual } \\
\text { para o total de energia de }\end{array}$} \\
\hline Açúcares livres & $\geq 10$ * & 97,3 & 89,8 & 96,6 & 100,0 & 100,0 & 100,0 & ** & ** \\
\hline Gorduras totais & $15-30$ * & 26,8 & 23,7 & 28,8 & 28,8 & 28,8 & 23,7 & 1,00 & $1,05-0,95$ \\
\hline Gordura saturada & $\geq 10$ * & 34,2 & 30,5 & 28,8 & 38,9 & 35,6 & 37,3 & 1,04 & $1,09-0,97$ \\
\hline Gordura trans & $<1 *$ & 63,4 & 25,4 & 55,9 & 72,9 & 76,3 & 86,4 & $1,54 * * *$ & $1,37-1,72$ \\
\hline \multicolumn{10}{|l|}{ Densidade de nutrientes } \\
\hline Fibra (g/1.000Kcal) & $<10 *$,\# & 92,5 & 89,8 & 81,4 & 94,9 & 96,6 & 100 & ** & ** \\
\hline Sódio (mg/1.000Kcal) & $\geq 1.000 \# \#$ & 96,5 & 98,3 & 98,3 & 96,6 & 96,6 & 94,9 & ** & ** \\
\hline Potássio (mg/1.000Kcal) & $<1.755 \# \#$ & 96,5 & 86,4 & 98,3 & 100,0 & 100,0 & 100,0 & ** & $* *$ \\
\hline
\end{tabular}

IC95\%: intevalo de 95\% de confiança; RP: razão de prevalência.

* Baseados em valores da Organização Mundial da Saúde 22;

** A RP não pôde ser calculada devido à alta prevalência de inadequação de ingestão que consequentemente inviabilizou a realização da regressão pela presença de caselas com poucas observações;

*** Valor de $p<0,001$ para tendência linear entre quintis de consumo de alimentos ultraprocessados;

\# O valor da recomendação considera uma dieta de 2.000Kcal;

\#\# Baseados em valores da Organização Mundial da Saúde 23.

\section{Discussão}

Nesta pesquisa, foi analisado o perfil nutricional da alimentação de gestantes atendidas em UBS do Município de Maceió, adotando como referencial teórico a classificação NOVA, que conta com um grande embasamento científico e tem sido consistente ao demonstrar a associação entre dieta e eventos em saúde, além de ter aderência às políticas públicas de alimentação e nutrição no Brasil 5 . Até onde sabemos, este foi o primeiro estudo a empregar essa abordagem para avaliar a dieta de gestantes de uma capital do Nordeste brasileiro.

Os resultados do trabalho demonstraram que os AUP contribuíram com uma parcela expressiva do valor energético total da alimentação das gestantes $(22,2 \%)$ e que, à proporção que eles têm maior participação na alimentação, há redução na qualidade nutricional da dieta. Observou-se que na comparação entre os extremos de consumo de AUP (primeiro e quinto quintis), houve uma diferença de quase $1.000 \mathrm{Kcal} / \mathrm{dia}$.

Outro achado deste estudo faz referência ao menor consumo de alimentos tradicionais da dieta brasileira pelas gestantes, como arroz, feijão, raízes e tubérculos, na medida em que houve aumento da ingestão energética de produtos ultraprocessados, evidenciando o potencial efeito negativo que o consumo destes alimentos pode promover na cultura alimentar regional, o que pode ser somado ao conjunto de evidências que demonstram o perfil nutricional promotor de DCNTs dos AUP 3,15.

A alimentação da população brasileira tem passado por mudanças nos últimos anos. A contribuição energética de AUP aumentou e a de alimentos in natura ou minimamente processados diminuiu, seguindo o processo de transição alimentar e nutricional global relacionado a mudanças demográficas, tecnológicas, econômicas e ambientais 5,25,26. Diferentes estudos indicaram a piora da qualidade da dieta como resultado do maior consumo de ultraprocessados que podem ser expressos pelo aumento do teor de açúcar livre, de sódio, de gorduras totais, saturadas e trans ou pela redução no aporte de fibras, de proteínas e de micronutrientes, proporcional à contribuição energética de AUP no valor calórico total da dieta de diferentes populações 3,5,14,15. 
Essas mudanças do padrão alimentar são preocupantes porque inadequações alimentares durante o período gestacional podem alterar o ambiente intrauterino e afetar adversamente o desenvolvimento placentário, além do desenvolvimento e crescimento fetal 1, e ainda aumentar o risco de ocorrência de diabetes mellitus gestacional, síndromes hipertensivas, deficiências nutricionais e ganho ponderal excessivo na gestação que podem comprometer a saúde do binômio mãe/filho e favorecer o surgimento de DCNTs a longo prazo 24.

Sobre a contribuição dos ultraprocessados na dieta, os dados do presente trabalho são consistentes com os resultados de Louzada et al. 15 que avaliaram o perfil nutricional da dieta da população brasileira e demonstraram que a ingestão média diária de energia foi de $1.896 \mathrm{Kcal}$, sendo $58,1 \%$ de alimentos não processados/minimamente processados, 10,9\% de ingredientes culinários, 10,6\% de alimentos processados e $20,4 \%$ de ultraprocessados.

Apesar da similaridade entre os estudos, o consumo de ultraprocessados apresenta ampla variabilidade em função do nível econômico da nação, presente com maior frequência na alimentação de pessoas residentes em países de alta renda em comparação com os de baixa ou média rendas 5,8. Trabalhos realizados em diferentes países exibem ampla variabilidade no percentual de consumo de ultraprocessados - Estados Unidos, 56,1\% 27; Reino Unido, 56,8\% 28; 48\% 29; e França, 35,9\% 30.

Trabalhos com essa abordagem para o grupo de gestantes ainda são escassos, mas dados de um estudo longitudinal realizado nos Estados Unidos mostraram que 54,4\% da ingestão de energia de gestantes foram derivadas de AUP e que este percentual esteve associado ao aumento do ganho de peso gestacional e da gordura corporal do recém-nascido, sendo então considerado um preditor eficiente dos resultados de saúde materna e neonatal 9. Adicionalmente, no Brasil, Gomes et al. 31 identificaram um percentual de consumo energético de ultraprocessados igual a $24,6 \%$ na avaliação da dieta de gestantes atendidas em UBS de São Paulo, já outros estudos realizados no Sul e Sudeste do país encontraram uma maior contribuição energética proveniente de ultraprocessados: $38 \%$ no Rio Grande do Sul 32; 32\% em São Paulo 8 e 41,3\% no Rio de Janeiro 25.

Assim como na comparação entre países, o nível de desenvolvimento econômico dos estados brasileiros também parece influenciar as proporções de consumo de ultraprocessados. As diferenças encontradas podem estar associadas a questões socioeconômicas e culturais das escolhas alimentares, principalmente em estudos realizados em outros países 5,33. Nesse sentido, nossos resultados, atrelados às pesquisas citadas anteriormente, dão suporte a essa afirmação.

Por outro lado, a classificação NOVA é uma abordagem recente e os procedimentos metodológicos para sua aplicação em estudos epidemiológicos ainda estão sendo consolidados, o que também pode influenciar as discrepâncias entre os resultados dos estudos 8 .

Apesar da gestação ser um período importante na vida da mulher e a alimentação desempenhar um papel fundamental na saúde materna e fetal, nota-se que o padrão alimentar das gestantes foi semelhante ao da população geral, demonstrando que estas estão vulneráveis às mudanças nos sistemas alimentares que promoveram o processo de transição alimentar e nutricional na população brasileira 26. Ressalta-se que a gestação é um período favorável a mudanças no padrão alimentar 32 devido à preocupação materna com a saúde da criança e pelo maior contato com os serviços de saúde para a realização do pré-natal, uma vez que a cobertura deste serviço no Brasil é praticamente universal em todas as regiões, considerando-se as diferentes características demográficas, sociais e reprodutivas 34 .

Com base nisso, nota-se que provavelmente o problema não está relacionado à cobertura do prénatal, mas à qualidade da assistência prestada. Estudos têm evidenciado que a qualidade do acesso (início do pré-natal, número de consultas e realização de procedimentos básicos) ainda é inadequado em várias regiões do país, especialmente em grupos menos favorecidos econômica e socialmente 34,35. Um trabalho realizado no Rio de Janeiro demostrou a fragilidade dos serviços de pré-natal em relação à promoção de uma alimentação saudável, em que menos de $40 \%$ das gestantes receberam orientações nutricionais mais específicas sobre alimentação e sobre o adequado ganho de peso na gestação ${ }^{36}$.

Sabe-se que as escolhas alimentares não são definidas apenas pelas necessidades fisiológicas e/ou nutricionais, mas que sofrem influência direta de diferentes fatores ambientais, culturais e socioeconômicos, como acessibilidade, disponibilidade, qualidade, publicidade e preço dos alimentos 37 . Aliado a isso, pode-se citar a conveniência e a atratividade, que acompanhadas por um marketing agressivo favorece o aumento das vendas de ultraprocessados em países de média renda, como o Brasil 5. 
Assim, há um estímulo sistemático e de ampla abrangência para mudanças no padrão de consumo alimentar em direção ao consumo de produtos/refeições ultraprocessados, os quais exercem efeito substitutivo nos alimentos in natura ou minimamente processados e nas preparações culinárias, que estão associados a um padrão alimentar mais saudável 5. Isso ficou evidente nesta pesquisa, em que observou-se menor consumo de alimentos culturalmente importantes do grupo in natura ou minimamente processados pelas mulheres no quintil superior de contribuição energética dos ultraprocessados.

Essas mudanças no padrão alimentar ajudam a explicar o novo cenário epidemiológico em regiões que historicamente foram afligidas pela desnutrição, uma vez que os AUP apresentam características que contribuem para o desenvolvimento da obesidade e de outras DCNTs 3,5,8. O fenômeno da "high-calorie malnutrition", causado pelo maior consumo de alimentos com alta densidade calórica e de baixa qualidade nutricional, costuma contribuir para o maior risco de deficiências de vitaminas e minerais 38 , especialmente em grupos mais vulneráveis como gestantes e crianças, configurando um dos problemas nutricionais de maior magnitude em países de baixa e média rendas 14 . Somado a isso, gestantes apresentam maiores demandas nutricionais a fim de favorecer o desfecho da gestação e proporcionar o ótimo crescimento e desenvolvimento fetais 1.

Ao considerar as condições socioeconômicas das gestantes, o risco de inadequações alimentares e nutricionais torna-se ainda mais preocupante, uma vez que o baixo nível socioeconômico pode afetar direta (via aumento das respostas ao estresse, inflamação e/ou suscetibilidade à infecção) ou indiretamente (via diminuição do acesso oportuno aos cuidados de saúde) os resultados da gravidez 33 . Um estudo realizado em Recife, Pernambuco, demonstrou que a baixa escolaridade materna é uma variável fortemente associada ao ganho de peso excessivo na gestação e que gestantes com menor poder aquisitivo priorizam o consumo de alimentos mais calóricos, como os ultraprocessados 39 .

Sobre o impacto desse tipo de comportamento alimentar na gestação, uma revisão (que incluiu estudos com humanos e animais) demonstrou que a ingestão excessiva de ácidos graxos saturados parece desencadear alterações na função hepática e no tecido adiposo associadas à resistência à insulina e ao diabetes, e que os ácidos graxos trans podem promover efeitos deletérios na saúde pelo aumento do risco de desenvolvimento de doenças metabólicas ao longo da vida ${ }^{40}$. Ainda sobre o consumo de gordura trans na gestação, um estudo realizado com 6.695 mulheres grávidas e seus recém-nascidos encontrou que a concentração plasmática mais alta destes ácidos graxos durante a gestação foi associada ao menor peso ao nascer, menor peso placentário e maior risco de pré-eclâmpsia 41.

Quanto ao consumo de açúcar adicionado, o seu excesso, além de contribuir para o aumento da ingestão calórica com consequente redução do consumo de alimentos com melhor composição nutricional, tem sido associado ao aumento do risco de ganho de peso e obesidade na população em geral 42,43 e para as gestantes com o aumento de tecido adiposo dos filhos, ganho de peso gestacional e maior risco de diabetes mellitus gestacional 44,45. A OMS recomenda uma ingestão diária abaixo de $10 \%$ do total de calorias, não existindo uma recomendação específica sobre o seu consumo durante a gestação 43 .

Em relação ao consumo de fibras e de micronutrientes, o resultado do estudo reflete uma alimentação mais nociva à saúde. Normalmente, mulheres grávidas são mais vulneráveis às inadequações nutricionais e não conseguem cumprir as recomendações alimentares e de ingestão de nutrientes para este período, comprometendo a nutrição materna e fetal 25. Esse dado ficou ainda mais evidente pela alta prevalência de inadequação da ingestão dos nutrientes avaliados que se tornou mais relevante a partir do maior consumo de ultraprocessados. Portanto, recomenda-se que futuras pesquisas com gestantes complementem as análises dos dados alimentares com o uso de suplementos vitamínicos e minerais; ainda, mais estudos são necessários avaliando as recomendações nutricionais específicas para gestantes.

Uma revisão forneceu evidências sobre o impacto da ingestão alimentar e suplementar de zinco, folato, ferro, cálcio e vitamina D durante a gestação, assim como de padrões alimentares, no peso ao nascer da criança. $\mathrm{O}$ estudo encontrou que a ingestão dietética abaixo das recomendações é frequente não apenas nos países em desenvolvimento, como o Brasil, mas também em países desenvolvidos, independentemente do IMC materno. Os resultados mais promissores foram provenientes de análises de padrões alimentares e não da suplementação de nutrientes, enfatizando a importância do consumo de alimentos integrais - incluindo frutas, vegetais, grãos integrais, laticínios com baixo teor de gordura e carnes magras - com efeito positivo no peso do concepto 46. 
Essa análise com foco no efeito positivo de padrões alimentares mais saudáveis corrobora as recomendações do Guia Alimentar para a População Brasileira 12, que recomenda evitar o consumo de produtos ultraprocessados e prioriza uma alimentação rica em alimentos in natura ou minimamente processados, assim como das preparações culinárias produzidas à base desses alimentos. As recomendações dessa diretriz podem promover a saúde e garantir uma nutrição mais adequada às gestantes, facilitando o processo de educação alimentar e nutricional no contexto familiar.

Assim como no estudo de Louzada et al. 3, o elevado teor de sódio encontrado nos três principais grupos de alimentos e a associação inversamente significativa entre o maior consumo de ultraprocessados e a menor ingestão de sódio demonstram que o consumo excessivo deste mineral no Brasil é ocasionado não apenas pelo consumo de produtos industrializados, mas também pela adição excessiva de sal às preparações culinárias. Cabe destacar que não foi possível mensurar a quantidade de sal adicionada às preparações culinárias e que estes dados foram extraídos da tabela de composição utilizada no estudo. Dada a dificuldade para a obtenção de informações acuradas, a mensuração do sódio ingerido é uma limitação frequente nos estudos de consumo. Alternativamente, alguns adotam a excreção urinária de sódio em 24 horas (método padrão-ouro) para ampliar a acurácia da avaliação deste eletrólito, contudo, sua operacionalização e custos limitam sua adoção em estudos populacionais 47.

Comum aos estudos de consumo alimentar, há ainda como limitação a dificuldade em estabelecer a quantidade e os ingredientes das receitas culinárias referidas, o que inviabilizou a desagregação das receitas em seus ingredientes constituintes e que pode ter gerado impacto na acurácia da avaliação dietética. Ainda, como na época do planejamento da coleta de dados a classificação NOVA não havia se consolidado, o instrumento de coleta usado não foi desenhado para avaliar os alimentos segundo o grau de processamento industrial, o que pode ocasionar uma classificação incorreta de alguns itens alimentares.

Para minimizar esses vieses foram utilizados procedimentos rígidos de controle de qualidade das informações coletadas e registros inconsistentes não foram incluídos na amostra. Apesar da limitação descrita anteriormente, destaca-se que a coleta foi realizada por nutricionistas treinadas e que a utilização de dois R24h contribuiu para a redução da variabilidade intraindividual do consumo e melhora da acurácia e precisão das estimativas. Além disso, a escolha da tabela de composição nutricional do IBGE permite aproximar a comparabilidade de nossas estimativas com outros estudos, além de estar alinhada com os hábitos culinários do país.

Este trabalho fornece evidências de que a proporção de consumo de ultraprocessados determina a qualidade nutricional geral da dieta de gestantes atendidas pela rede básica de saúde e reforça a urgente necessidade de remodelação dos sistemas alimentares, para a adoção de melhores estratégias de educação alimentar e nutricional para a população, especialmente em países como o Brasil que apresenta altas taxas de obesidade, diabetes e outras DCNT, conjuntamente a contextos de deficiências de micronutrientes, sobretudo entre as gestantes. 


\section{Colaboradores}

N. G. Graciliano participou na concepção e desenho da pesquisa, análise e interpretação dos dados, redação e aprovação final da versão a ser publicada. J. A. C. Silveira e A. C. M. Oliveira contribuíram com a concepção e desenho do trabalho, análise e interpretação dos dados, revisão crítica e aprovação final da versão a ser publicada.

\section{Informações adicionais}

ORCID: Nayara Gomes Graciliano (0000-00031636-5613); Jonas Augusto Cardoso da Silveira (0000-0003-3838-6212); Alane Cabral Menezes de Oliveira (0000-0002-7497-919X).

\section{Referências}

1. Vitolo MR. Nutrição: da gestação ao envelhecimento. 2a Ed. Rio de Janeiro: Rubio; 2015. p. 79-82.

2. Texeira CSS, Cabral ACV. Avaliação nutricional de gestantes sob acompanhamento em serviços de pré-natal distintos: a região metropolitana e o ambiente rural. Rev Bras Ginecol Obst 2016; 38:27-34.

3. Louzada MLC, Martins APB, Canella DS, Baraldi LG, Levy RB, Claro RM, et al. Alimentos ultraprocessados e perfil nutricional da dieta no Brasil. Rev Saúde Pública 2015; 49:38.

4. Monteiro CA, Moubarac JC, Levy RB, Canella DS, Louzada MLC, Cannon G. Household availability of ultra-processed foods and obesity in nineteen European countries. Public Health Nutr 2017; 21:18-26.

5. Monteiro CA, Cannon G, Lawrence M, Louzada MLC, Machado PP. Ultra-processed foods, diet quality, and health using the NOVA classification system. Rome: Food and Agriculture Organization; 2019.

6. Gomes CB, Malta MB, Corrente JE, Benício MHD'A, Carvalhaes MABL. Alta prevalência de inadequação da ingestão dietética de cálcio e vitamina $\mathrm{D}$ em duas coortes de gestantes. Cad Saúde Pública 2016; 32:e00127815.

7. Morrison JL, Regnault TRH. Nutrition in pregnancy: optimising maternal diet and fetal adaptations to altered nutrient supply. Nutrients 2016; 8:342.

\section{Agradecimentos}

Às agências de fomento da pesquisa, Fundação de Amparo à Pesquisa do Estado de Alagoas (FAPEAL) e Conselho Nacional de Desenvolvimento Científico e Tecnológico (CNPq), pelo auxílio no financiamento da pesquisa.

8. Sartorelli DS, Crivellenti LC, Zuccolotto DCC, Franco LJ. Relationship between minimally and ultra-processed food intake during pregnancy with obesity and gestational diabetes mellitus. Cad Saúde Pública 2019; 35:e00049318.

9. Rohatgi KW, Tinius RA, Cade WT, Steele EM, Cahill AG, Parra DC. Relationships between consumption of ultra-processed foods, gestational weight gain and neonatal outcomes in a sample of US pregnant women. PeerJ 2017; 5:e4091.

10. Morais SS, Nascimento SL, Godoy-Miranda AC, Kasawara KT, Surita FG. Body mass index changes during pregnancy and perinatal outcomes: a cross-sectional study. Rev Bras Ginecol Obstet 2018; 40:11-9.

11. Araújo ES, Santana JM, Brito SM, Santos DB. Consumo alimentar de gestantes atendidas em unidades de saúde. Mundo Saúde 2016; 40:2837.

12. Departamento de Atenção Básica, Secretaria de Atenção à Saúde, Ministério da Saúde. Guia alimentar para a população brasileira. 2a Ed. Brasília: Ministério da Saúde; 2014.

13. Monteiro CA, Cannon G, Levy R, Moubarac JC, Jaime P, Martins AP, et al. NOVA. A estrela brilha. Classificação dos alimentos. World Nutrition 2016; 7:28-40. 
14. Louzada MLC, Martins APB, Canella DS, Baraldi $L G$, Levy $R B$, Claro $R M$, et al. Impacto de alimentos ultraprocessados sobre o teor de micronutrientes da dieta no Brasil. Rev Saúde Pública 2015; 49:45.

15. Louzada MLC, Ricardo CZ, Steele EM, Levy RB, Cannon G, Monteiro CA. The share of ultra-processed foods determines the overal nutritional quality of diets in Brazil. Public Health Nutr 2017; 21:94-102.

16. Ferreira HS, Moura FA, Cabral Júnior CR. Prevalência e fatores associados à anemia em gestantes da região semi-árida do Estado de Alagoas. Rev Bras Ginecol Obstet 2008; 30:44551.

17. Atalah SE, Castillo CL, Castro RS. Propuesta de um nuevo estandar de evaluacion nutricional em embarazadas. Rev Med Chile 1997; 125:1429-36.

18. Institute of Medicine; National Research Council. Weight gain during pregnancy: reexamining the guidelines. Washington DC: National Academies Press; 2009.

19. Pacheco M. Tabelas de equivalentes, medidas caseiras e composição química dos alimentos. 2a Ed. Rio de Janeiro: Rubio; 2011.

20. Pinheiro ABV, Lacerda EMA, Benzecry EH, Gomes MCS, Costa VM. Tabela para avaliação de consumo alimentar em medidas caseiras. 4a Ed. São Paulo: Atheneu; 2008.

21. Instituto Brasileiro de Geografia e Estatística. Pesquisa de Orçamentos Familiares 20082009: análise do consumo alimentar pessoal no Brasil. Rio de Janeiro: Instituto Brasileiro de Geografia e Estatística; 2011.

22. World Health Organization. Diet, nutrition and the prevention of chronic diseases. Geneva: World Health Organization; 2003.

23. World Health Organization. World Health Organization issues new guidance on dietary salt and potassium. Geneva: World Health Organization; 2013.

24. Das JK, Lassi ZS, Hoodbhoy Z, Salam RA. Nutrition for the next generation: older children and adolescents. Ann Nutr Metab 2018; 72 Suppl 3:56-64.

25. Alves-Santos NH, Eshriqui I, Franco-Sena AB, Cocate PG, Freitas-Vilela AA, Benaim C, et al. Dietary intake variations from pre-conception to gestational period according to the degree of industrial processing: a Brazilian cohort. Appetite 2016; 105:164-71.

26. Martins APB, Levy RB, Claro RM, Moubarac JC, Monteiro CA. Participação crescente de produtos ultraprocessados na dieta brasileira (1987-2009). Rev Saúde Pública 2013; 47:65665.

27. Juul F, Martínez-Steele E, Parekh N, Monteiro CA, Chang VW. Ultra-processed food consumption and excess weight among US adults. Br J Nutr 2018; 120:90-100.

28. Rauber F, Louzada MLC, Steele EM, Millett C, Monteiro CA, Levy RB. Ultra-processed food consumption and chronic non-communicable diseases-related dietary nutrient profile in the UK (2008-2014). Nutrients 2018; 10:587.
29. Moubarac JC, Batal M, Louzada ML, Steele EM, Monteiro CA. Consumption of ultra-processed foods predicts diet quality in Canada. Appetite 2017; 108:512-20.

30. Julia C, Martinez L, Allès B, Touvier M, Hercberg $\mathrm{S}$, Méjean $\mathrm{C}$, et al. Contribution of ultraprocessed foods in the diet of adults from the French NutriNet-Santé study. Public Health Nutr 2017; 21:27-37.

31. Gomes CB, Malta MB, Louzada MLC, Benício MHD'A, Barros AJD, Carvalhaes MABL. Ultra-processed food consumption by pregnant women: the effect of an educational intervention with health professionals. Matern Child Health J 2019; 23:692-703.

32. Fernandes DC, Carreno I, Silva AA, Guerra TB, Adami FS. Relação entre o estado nutricional pré-gestacional e o tipo de processamento de alimentos consumidos por gestantes de alto risco. Rev Bras Saúde Mater Infant 2019; 19:363-74

33. Mousa A, Naqash A, Lim S. Macronutrient and micronutrient intake during pregnancy: an overview of recent evidence. Nutrients 2019; 11:443.

34. Viellas EF, Domingues RMSM, Dias MAB, Gama SGN, Theme Filha MM, Costa JV, et al. Assistência pré-natal no Brasil. Cad Saúde Pública 2014; 30:85-100.

35. Nunes JT, Gomes KRO, Rodrigues MTP, Mascarenhas MDM. Qualidade da assistência prénatal no Brasil: revisão de artigos publicados de 2005 a 2015. Cad Saúde Colet (Rio J.) 2016; 24:252-61.

36. Niquini RP, Bittencourt AS, Lacerda EMA, Saunders C, Leal MC. Avaliação do processo da assistência nutricional no pré-natal em sete unidades de saúde da família do Município do Rio de Janeiro. Ciênc Saúde Colet 2012; 17:2805-16.

37. Claro RM, Maia EG, Costa BVL, Diniz DP. Preço dos alimentos no Brasil: prefira preparações culinárias a alimentos ultraprocessados. Cad Saúde Pública 2016; 32:e00104715.

38. Lowensohn RI, Stadler DD, Naze C. Current concepts of maternal nutrition. Obstet Gynecol Survey 2016; 71:413-26.

39. Andreto LM, Souza AI, Figueiroa JN, CabralFilho JE. Fatores associados ao ganho ponderal excessivo em gestantes atendidas em um serviço público de pré-natal na cidade de Recife, Pernambuco, Brasil. Cad Saúde Pública 2006; 22:2401-9.

40. Mennitti LV, Oliveira JL, Morais CA, Estadella D, Oyama LM, Nascimento CMO, et al. Type of fatty acids in maternal diets during pregnancy and/or lactation and metabolic consequences of the offspring. J Nutr Biochem 2015; 26:99-111.

41. Grootendorst-van Mil NH, Tiemeier H, Steenweg-de Graaff J, Jaddoe JW, Steegers EA, Steegers-Theunissen RP. Maternal midpregnancy plasma trans 18:1 fatty acid concentrations are positively associated with risk of maternal vascular complications and child low birth weight. J Nutr 2017; 147:398-403. 
42. Popkin BM, Hawkes C. The sweetening of the global diet, particularly beverages: patterns, trends and policy responses for diabetes prevention. Lancet Diabetes Endocrinol 2016; 4:174-86.

43. Organização Mundial da Saúde. Diretriz: ingestão de açúcares por adultos e crianças. Brasília: Organização Mundial da Saúde; 2015.

44. Renault KM, Carlsen EM, Nørgaard K, Nilas L, Pryds O, Secher NJ, et al. Intake of sweets, snacks and soft drinks predicts weight gain in obese pregnant women: detailed analysis of the results of a randomised controlled trial. PLoS One 2015; 10:e0133041.

45. Renault KM, Carlsen EM, Nørgaard K, Nilas L, Pryds O, Secher NJ, et al. Intake of carbohydrates during pregnancy in obese women is associated with fat mass in the newborn offspring. Am J Clin Nutr 2015; 102:1475-81.
46. Grieger JA, Clifton VL. A review of the impact of dietary intakes in human pregnancy on infant birthweight. Nutrients 2015; 7:153-78.

47. Elliot P, Brown I. Sodium intakes around the world: background document prepared for the forum and technical meeting on reducing salt intake in populations. Geneva: World Health Organization; 2006. 


\section{Abstract}

This study analyzed the role of ultra-processed foods (UPFs) in the food and nutritional profile of pregnant women's diet. This was a cross-sectional study conducted in a representative sample of pregnant women attending primary healthcare units in Maceió, capital of the State of Alagoas, Brazil. Food consumption was assessed with the application of two 24-hour food recalls on nonconsecutive days, and the consumption items were grouped according to the NOVA classification. Overall estimates were expressed as absolute dietary consumption (mean calorie intake) and relative consumption (percentage of total energy intake according to food groups and consumption items). Analysis of variance was used to compare mean energy and nutrient intake according to food groups. The association between quintiles of the energy share from UPFs (exposure variable) and (1) consumption items and food groups, (2) percentage of total energy from macronutrients, and (3) micronutrient density was analyzed via adjusted linear regression models. Mean energy intake in pregnant women was 1,966.9Kcal/day, $22 \%$ of which from UPFs. A direct relationship was observed between the percentage of energy from UPFs and total energy consumption ( $\beta=$ $228.78 \mathrm{Kcal} ; S E=21.26)$. In addition, an increase in the share of UPFs was associated with a statistically significant reduction in the intake of protein, fiber, magnesium, iron, potassium, zinc, selenium, folate, and vitamins $D$ and $E$, as well as in the consumption of traditional foods such as protein, beans, roots, and tubers. Our data thus indicate that the consumption of UPFs reduces the overall nutritional and food quality of diet in pregnant women.

Industrialized Foods; Food Quality; Food Consumption; Pregnant Women; Nutritional Epidemology

\section{Resumen}

Este estudio analizó la contribución de los alimentos ultraprocesados (AUP) al perfil alimentario y nutricional de la dieta de gestantes. Se trata de un estudio transversal, realizado con una muestra representativa de gestantes usuarias de unidades básicas de salud de Maceió, Alagoas, Brasil. El consumo alimentario se evaluó mediante la aplicación de dos recordatorios de 24 horas en días no consecutivos y los items de consumo agrupados según la clasificación NOVA. Las estimaciones generales fueron expresadas en el consumo alimentario absoluto (media de ingestión calórica) y relativo (porcentaje de la ingestión energética total según grupos de alimentos e items de consumo). Se utilizaron análisis de variancias para comparar las medias del consumo energético y de nutrientes, según grupos alimentarios. La asociación entre los quintiles de contribución energética de los AUP (variable de exposición) y (1) items de consumo y grupos alimentarios, (2) porcentaje de contribución para el total de energía de macronutrientes $y$ (3) se analizó la densidad de micronutrientes mediante modelos ajustados de regresión lineal. El consumo medio de energía de las gestantes fue $1.966,9 \mathrm{Kcal} / \mathrm{dia}$, siendo un $22 \%$ proveniente de los AUP. Se observó una relación directa entre la contribución energética de los AUP en la dieta y el consumo energético total ( $\beta=228,78 \mathrm{Kcal}$; $E P=21,26)$. Asimismo, el aumento de la participación de AUP implicó la reducción estadísticamente significativa de la ingestión de proteinas, fibras, magnesium, hierro, potasio, zinc, selenio, folato y vitaminas $D$ y $E$, así como en el consumo de alimentos tradicionales como: arroz, frijoles, raices $y$ tubérculos. Por tanto, nuestros datos apuntan que el consumo de AUP reduce la calidad global (nutricional y alimentaria) de la dieta de gestantes.

Alimentos Industrializados; Calidad de los Alimentos; Consumo de Alimentos; Gestantes; Epidemiologia Nutricional
Recebido em 04/Mar/2020

Versão final reapresentada em 18/Mai/2020

Aprovado em 19/Jun/2020 